\begin{tabular}{|c|c|}
\hline Title & Polymer A dsorbed Bilayer Membranes Form Self-Healing Hydrogels with Tunable Superstructure \\
\hline Author(s) & $\begin{array}{l}\text { Li, X ufeng; Kurokawa, Takayuki; T akahashi, Riku; Haque, Md. A namul; Y ue, Y oufeng; Nakajima, Tasuku; Gong, Jian } \\
\text { Ping }\end{array}$ \\
\hline Citation & $\begin{array}{l}\text { Macromolecules, 48(7), 2277-2282 } \\
\text { https://doi.org/10.1021/acs.macromol.5b00422 }\end{array}$ \\
\hline Issue Date & $2015-04-14$ \\
\hline Doc URL & http:/hdl.handle.net/2115/61108 \\
\hline Rights & $\begin{array}{l}\text { This document is the A ccepted Manuscript version of a Published Work that appeared in final form in Macromolecules, } \\
\text { copyright @ A merican Chemical Society after peer review and technical editing by the publisher. To access the final } \\
\text { edited and published work see http://pubs.acs.org/doi/abs/10.1021/acs.macromol.5b00422. }\end{array}$ \\
\hline Type & article (author version) \\
\hline File Information & manuscript_Li .pdf \\
\hline
\end{tabular}

Instructions for use 


\section{Polymer Adsorbed Bilayer Membranes Form Self- healing Hydrogels with Tunable Superstructure}

Xufeng Li $i^{\dagger}$, Takayuki Kurokawa ${ }^{\dagger}$, Riku Takahashi ${ }^{\dagger}$, Md. Anamul Haque ${ }^{\dagger, \S}$, Youfeng Yu ${ }^{\dagger, \S}$, Tasuku Nakajima ${ }^{t}$, and Jian Ping Gong ${ }^{*}+\dot{t}$

${ }^{\dagger}$ Graduate School of Life Science, and Faculty of Advanced Life Science, Hokkaido University, Sapporo 060-0810, Japan

ABSTRACT: We report that polymers can support bilayer membranes to form physical hydrogels of self-healing and tunable isotropic/anisotropic structure. The system consists of poly(dodecyl glyceryl itaconate) (PDGI) that forms lamellar bilayers and polyacrylamide (PAAm) that adsorbs on the bilayer surfaces via hydrogen bond formation. Adsorption of PAAm brings two effects: disturbs the bilayer packing and causes bending of the bilayers; increases the effective thickness of the bilayers and enhances the repulsion between the bilayers due to excluded volume effect. Competition of these two effects brings about sharp super-structure transition from isotropic multi-layer foam phase to unidirectionally aligned lamellar phase. Accompany with this structure transition, the bulk hydrogel exhibits isotropic/anisotropic swelling. The physical gels exhibit high tensile strength and self-healing properties that can be understood by the sacrificial bonds mechanism. 


\section{INTRODUCTION}

Conventional hydrogels are isotropic in structure and weak in mechanical strength. Recent studies on double network hydrogels have shown that by introducing rigid and brittle sacrificial bonds to soft and ductile matrix, the hydrogels become tough by dissipate energy during deformation. ${ }^{1-4}$ The innovative developments of tough hydrogels have greatly expanded the potential applications of this material in various fields, including soft actuators, artificial organs, regenerative medicine, etc. ${ }^{5-12}$ For these potential use, the next challenge is to develop tough hydrogels with anisotropic super-structures of macroscopic scale, as like biological tissues. ${ }^{13} \mathrm{~A}$ promising strategy is to incorporate self-assembled structures into hydrogels. The self-assembled structures, usually being more rigid and brittle than polymer networks, can rupture at loading and reform at unloading. Therefore, they not only impart various anisotropic properties, but also have potential as reversible sacrificial bonds to make the soft materials tough and self-healing.

Directional molecules, for example, lipid molecules, can self-assemble into various mesoscale super-structures ${ }^{14-23}$ in aqueous solution, notably lamellar structure and vesicles. However, usually it is very difficult to build these meso-scale structures into the macroscopic scale, especially in hydrogels. Some recent works have succeeded in developing hydrogels with macroscale super-structures. ${ }^{24-27}$ An interesting example is the dodecyl glyceryl itaconate/acrylamide (DGI/AAm) system..$^{28-31}$ The gel, consisting of poly(dodecyl glyceryl itaconate) (PDGI) that form lamellar bilayers and chemically cross-linked polyacrylamide (PAAm), exhibits many unique functions that are substantially superior to conventional hydrogels, such as structure color, unidirectional swelling, high toughness, and self-healing. Despite these excellent functions, the structure formation mechanism of bilayers and the interaction between the bilayer and the polymer in the hydrogel are still unknown. Considering the vulnerable nature of the bilayer 
membranes that are easily disturbed by subtle changes in the environment conditions, it is an interesting puzzle why bilayers form macro-scale, mono-domain lamellar structure in PAAm hydrogel.

Herein, we intend to elucidate the mechanism of the PDGI bilayer super-structure formation in PAAm hydrogels. To reveal the interaction between PDGI bilayer and PAAm, we focus on the linear polymer system without using any chemical cross-linker. We systematically study the structure evolution of the precursor monomer solution, the as-prepared gel, and waterequilibrated gel (Figure 1). We elucidated, for the first time that, the PAAm chains adsorb on the bilayer surfaces via hydrogen bond to form physical hydrogels. The adsorption of polymer causes the isotropic/anisotropic bilayer structure transition. Corresponding to this structure change, the gels showed unique swelling behaviors. Furthermore, the physical gels showed very large stretchability and self-healing properties.

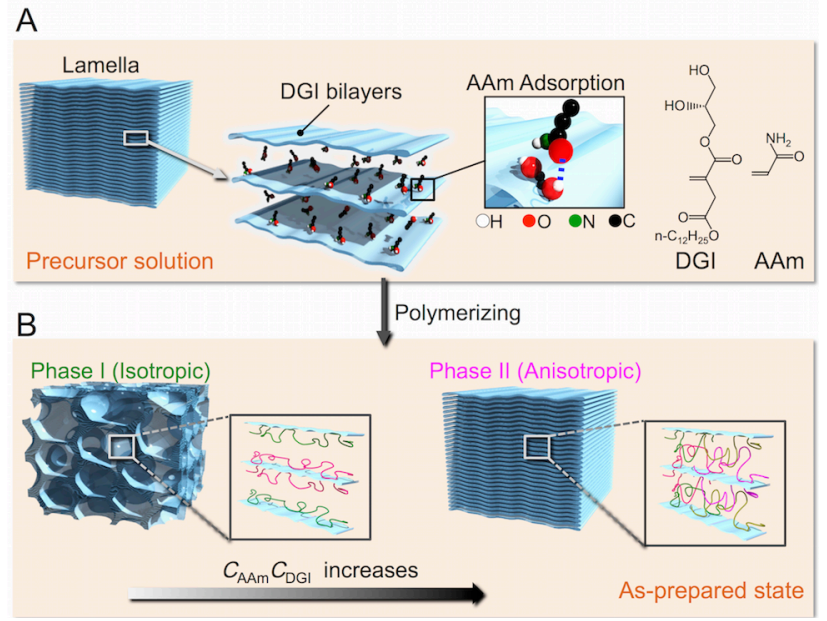

Figure 1. Schematic diagram of the structure in (A) precursor solution and (B) as-prepared gels.

\section{RESULTS AND DISCUSSION}


Lipid monomer DGI self-assembles into lamellar structure in water, as confirmed by the iridescent color of the sample due to the Bragg reflection. ${ }^{32}$ The addition of AAm $(0.01 \sim 3.0 \mathrm{M})$ does not destroy the lamellar structure, although AAm adsorbs on the surface of bilayers by hydrogen bond formation (Figure 1A), as revealed by the Fourier transform infrared (FTIR) spectroscopy (Figure S1). After polymerization, physical hydrogels were formed. As the DGI and AAm have been found to form homopolymers even by one-step polymerization, ${ }^{33}$ the hydrogel formation is associated to the hydrogen bonding between PDGI bilayer and PAAm, which served as physical cross-linking. This is confirmed by the phenomena that the sole DGI solution and the sole AAm solution do not form hydrogels after polymerization.
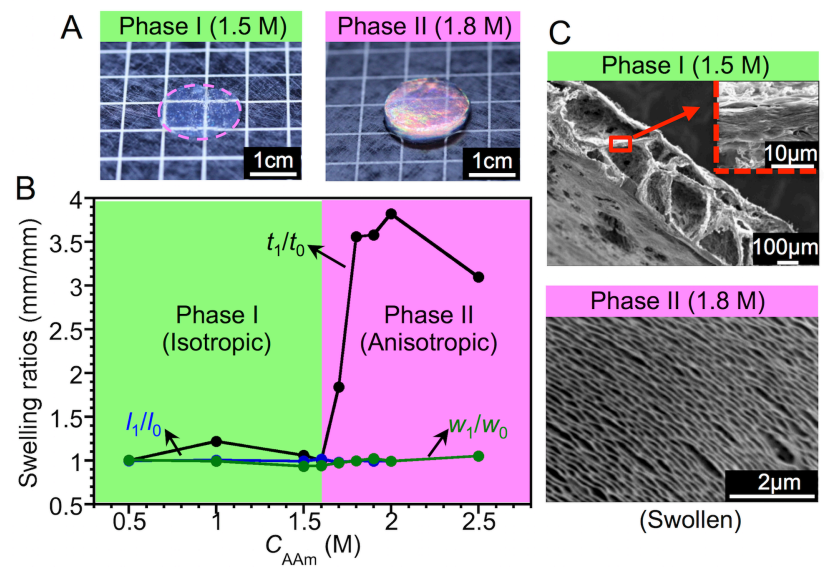

Figure 2. Appearance, swelling behavior, and structures of the PDGI/PAAm hydrogels in phase I and II. (A) Appearance of the swollen gels. (B) Relationship between $C_{\mathrm{AAm}}$ and the swelling ratio of PDGI/PAAm physical hydrogels in thickness $\left(t_{1} / t_{0}\right)$, length $\left(l_{1} / l_{0}\right)$ and width $\left(w_{1} / w_{0}\right)$ in water relative to the as-prepared gels. (C) Typical cross-section SEM images of the swollen gels with different $C_{\mathrm{AAm}}$ in different phases. In phase I and II, vesicle structure and lamellar structure are maintained after swelling. For all of the samples, $C_{\mathrm{DGI}}=0.1 \mathrm{M}$, numbers in (A) and (C) are $C_{\mathrm{AAm}}$. 
The hydrogels show two phases when change the concentration of AAm $C_{\mathrm{AAm}}$ but keep the DGI concentration $C_{\mathrm{DGI}}$ constant $(0.1 \mathrm{M})$, as revealed by the appearance (Figure $2 \mathrm{~A}$ ) and unique swelling behaviors (Figure $2 \mathrm{~B})$. In phase $\mathrm{I}\left(C_{\mathrm{AAm}} \leq 1.6 \mathrm{M}\right)$, the samples were transparent, colorless, and hardly swelled. Large porous structure was observed by naked eyes. In phase II (1.6 $\left.\mathrm{M}<C_{\mathrm{AAm}} \leq 3.0 \mathrm{M}\right)$, samples showed iridescent color, and swelled abruptly in thickness direction but not in length and width directions. Accompanying with this anisotropic swelling, red shift in the color was observed. These results suggest a unidirectionally aligned lamellar structure in phase II. From the Bragg's reflection spectra, the lamellar distance shifts from the asprepared $115 \mathrm{~nm}$ to $461 \mathrm{~nm}$ after swelling for $C_{\mathrm{AAm}}=1.8 \mathrm{M}$ (Figure 2B). The anisotropic swelling in thickness direction remained but became less prominent with increase in $C_{\mathrm{AAm}}$.
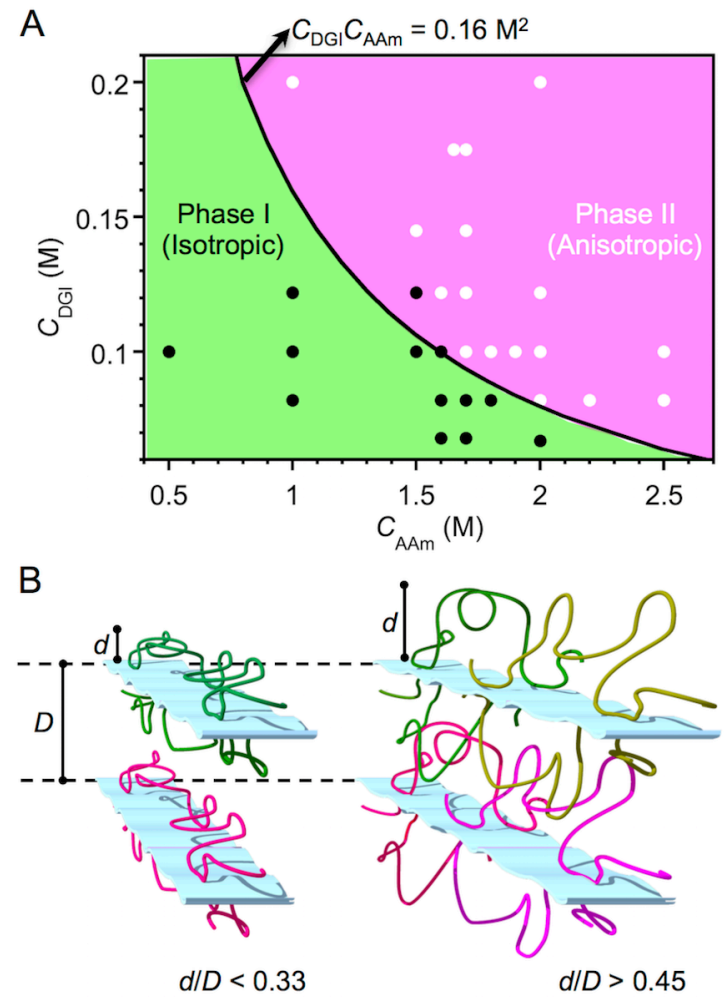

Figure 3. Phase diagram and isotropic/anisotropic phase transition mechanism. (A) Phase diagram of PDGI/PAAm physical hydrogels classified by swelling behavior and structure 
analysis. The phase I/II boundary follows a relationship of $C_{\mathrm{DGI}} C_{\mathrm{AAm}}=0.16 \mathrm{M}^{2}$. (B) Excluded volume explanation for the isotropic/anisotropic structure transition with increase in the ratio of the adsorbed bilayer thickness $\mathrm{d}$ to the lamellar distance $D$.

The hydrogel structures are further characterized by polarizing optical microscopy (POM) and scanning electron microscopy (SEM) for the samples before and after swelling. In phase I, the as-prepared gels showed almost no or weak birefringence in the side view of POM, and the weak birefringence completely disappeared after the samples being immersed in water (Figure S2). Large irregular pores, of $\sim 100 \mu \mathrm{m}$ in diameter, were observed in POM observation and SEM observation (Figure S3 and Figure S4). The pore size increased with an increase in $C_{\mathrm{AAm}}$. Multilayers, related to the stacking of PDGI bilayers, were identified on the edge of pores, indicating the multilayer foam structure. The non-swelling behavior in phase I indicates that the multilayer forms continuous phase to completely constrain the swelling, since the bilayers are non-permeable to water whereas PAAm intends to swell in water. This explains why we still observed the foam structure even after immersing the samples in water (Figure 2C).

In phase II, the as-prepared gels showed strong birefringence from the side view (Figure S2). This birefringence is related to the well-ordered lamellar structure (parallel to the sample surface) as confirmed by SEM observation (Figure 2C). This indicates that the gels are composed of the alternative stacking of rigid, water-impermeable PDGI layers and soft, waterswellable PAAm layers (Figure 1B). After being immersed in water, the anisotropic lamellar structure is maintained, confirmed by the strong birefringence of POM observation and SEM observation (Figure S2 and Figure 2C). This unidirectionally aligned lamellar structure well explains why the gels only swelled in thickness direction perpendicular to the bilayers. That is, the PDGI bilayers, impermeable to water, restrain the swelling of PAAm along the bilayer 
direction. This result suggests that the hydrogen bonds between PDGI layers and PAAm layers are strong enough to prevent desorption caused by swelling mismatching between bilayer and polymer.

Physical PDGI/PAAm hydrogels can be formed in the range of the $C_{\mathrm{AAm}}(0.01 \sim 3.0 \mathrm{M})$ and $C_{\mathrm{DGI}}(0.04 \sim 0.20 \mathrm{M})$, although the gels with low $C_{\mathrm{DGI}}$ are so weak that they were broken during swelling. The phase I/II boundary was found to shift to a lower critical $C_{\mathrm{AAm}}\left(C_{\mathrm{AAm} \text {,ritical }}\right)$ with an increase in $C_{\text {DGI }}$ (Figure S5). Thus, a concentration phase diagram is constructed (Figure 3A), and we found that the phase I/II boundary follows a curve with the relation of $C_{\mathrm{DGI}} C_{\mathrm{AAm}}=0.16$ $\mathrm{M}^{2}$, where isotropic phase I is below the curve and anisotropic phase II is above the curve, indicating the coupled influence of DGI and AAm on the structure transition.

The physical gels in phase I were completely soluble in DMSO that is a good solvent for both PDGI and PAAm, indicating that the hydrophobic association between PDGI, which may lead to the gelation by connect the neighboring foams, were destroyed by DMSO. In phase II, the gel swelled substantially but not dissolved completely in DMSO, indicating that the hydrogen bonds between bilayers and PAAm are very strong (Figure S6).

From the above results, we can discuss the mechanism of the phase I/II structure transition. Without AAm, the lamellar bilayer structure is maintained even after polymerization. With a small amount of AAm, the adsorption of the PAAm chains on the surface of bilayers disturbs the structure of the bilayers. As a result, the bilayers make bending to form multilayer foam structure (phase I, Figure 1B). As the multilayer foams, which are water impermeable, forms a continuous structure, the gel does not swell in water. With the increase in $C_{\mathrm{AAm}}$, more PAAm chains are adsorbed to form thick layers. ${ }^{34-38}$ Each PAAm adsorbed bilayer behaves as a thick platelet 
(Figure 3B), and when the volume fraction of these thick platelets is high enough, the layer could not bend and maintain the lamellar structure due to the excluded volume effect (steric repulsion) between the adjacent platelets (phase II, Figure 1B). As a result, the adsorption of thick PAAm layers stabilizes the lamellar structure.

We try to correlate the observed structure transition condition $C_{\mathrm{DGI}} C_{\mathrm{AAm}}=$ constant to the excluded volume effect using the Onsager's mean-field theory. ${ }^{39,40}$ According to the theory, for a platelet system, the ratio of the thickness of one platelet to the lamellar distance is the key parameter to determine the stability of the anisotropic lamellar structure, which can be simply expressed as $d / D$ in this system, where $d$ is the adsorbed polymer thickness and $D$ is the lamellar distance (Figure 3B). When $d / D<0.33$, the platelets have enough space to move, so the lamellar structure is unstable and tends to form isotropic arrangement; when $d / D>0.45$, the platelets are restricted to form stable anisotropic structure. From this theory, the region of $0.33<d / D<0.45$ is a transition region. Since the lateral size of the bilayer membranes is much larger than $D$ (several hundreds of nanometers), we assume that the present system meets this mean-field theory. Previous study has shown that $D$ deceases with an increase in $C_{\mathrm{DGI}}$, that is, $C_{\mathrm{DGI}} \sim 1 / D .^{32}$ Furthermore, literature tells that the polymer adsorption on surfaces has a self-similar feature. ${ }^{34}$ At low $C_{\mathrm{AAm}}$, bilayer surface are unsaturated with adsorbed polymers (flat conformation), thus d is small, in the order of monomer size. When the polymer concentration reach a critical value $C_{\mathrm{AAm}, \mathrm{c}}$, the adsorption is saturated to monolayer. Above $C_{\mathrm{AAm}, \mathrm{c}}$, meshes, loops, and tails develop, and $\mathrm{d}$ increases abruptly and approaches to the radius of gyration of the polymer chain $R_{\mathrm{F}} \cdot{ }^{36}$ The saturated adsorption, $C_{\mathrm{AAm}, \mathrm{c}}$, is equal to $C_{\mathrm{DGI}}\left(<<C_{\mathrm{AAm}}\right)$ at most. If we simply assume that $\mathrm{d}$ increases linearly with $C_{\mathrm{AAm}}-C_{\mathrm{AAm}, \mathrm{c}} \approx C_{\mathrm{AAm}}$ above the critical $C_{\mathrm{AAm}, \mathrm{c}}$, then the product of the two monomer concentrations $C_{\mathrm{AAm}} C_{\mathrm{DGI}}$ is linearly proportional to $d / D$. That is, $C_{\mathrm{AAm}} C_{\mathrm{DGI}} \sim d / D$. This 
well explains why the boundary between phase I and II (Figure 3A) is governed by a curve of $C_{\mathrm{AAm}} C_{\mathrm{DGl}}=$ constant.

To form physical gels in phase II, PAAm chains should serve as crosslinker to bridge neighbouring bilayers. The physical cross-linking could be formed by two mechanisms: the topological entanglement of PAAm chains adsorbed onto different bilayers, and the bridging between neighbouring bilayers by a single PAAm chain. Both of the mechanisms should occur at $d / D>0.5$. This condition is stricter than but close to the condition for isotropic/anisotropic structure transition $d / D>0.45$. This explains why we observed weak and highly swollen gels right above the I/II structure transition curve. Considering the gelation condition, the boundary $C_{\mathrm{AAm}} C_{\mathrm{DGI}}=0.16 \mathrm{M}^{2}$ should correspond to $d / D=0.5$, above which the lamellar structure can be directly crosslinked by the PAAm entanglement.

The swelling ratio $t_{1} / t_{0}$ in phase II is determined by the balance of the osmotic pressure of the adsorbed polymer layer and the elasticity of physical cross-linking. The former is proportional to $C_{\mathrm{AAm}}{ }^{9 / 4}{ }^{41}$ while the physical cross-linking density increases with the $C_{\mathrm{AAm}}$. The sharp increase in the swelling ratio in thickness direction just above the transition point indicates that the osmotic pressure term is dominant in this region and then the bridging density and entanglement density dramatically increase with $C_{\mathrm{AAm}}$, leading to a suppression of the swelling ratio with increase in $C_{\mathrm{AAm}}$ (Figure 2B and Figure S5). When $C_{\mathrm{DGI}}$ is very low, the chain bridging and entanglement is suppressed, so the swelling ratio only increases with $C_{\mathrm{AAm}}$ (Figure S5). At a very high $C_{\mathrm{AAm}}(>3$ $\mathrm{M}$ ), the strong osmotic pressure of PAAm broke the hydrophobic association, so the gels showed an isotropic swelling behavior. 
It should be noted that the maximum lamellar distance $D$ that is possible to form lamellar phase gel (phase II) is related to the maximum adsorption thickness $d_{\max }$ of polymer. $d_{\max }$ is equal to the radius of gyration of the polymer, $R_{\mathrm{F}}$. When $R_{\mathrm{F}} / D<0.45$, no lamellar phase gel could be formed no matter how high the $C_{\mathrm{AAm}}$ is. For the present system, the molecular weight of PAAm is $\sim 10^{6} \mathrm{~g} / \mathrm{mol}$, we have $d_{\max }=R_{\mathrm{F}}=53 \mathrm{~nm} \cdot{ }^{36}$ For $C_{\mathrm{DGI}}=0.1 \mathrm{M}, D$ estimated from Brag diffraction of the as-prepared sample is $115 \mathrm{~nm}$. This gives $R_{\mathrm{F}} / D=0.46$. So at $C_{\mathrm{DGI}}=0.1 \mathrm{M}, D$ is very close to the maximum inter-bilayer distance to form a lamellar structure gel with PAAm.

The above revealed structure formation mechanism applies to the PDGI/PAAm chemical hydrogels that also showed anisotropic structure. ${ }^{28}$ Addition of chemical cross-linker brings an effect to increase the effective PAAm chain length and promote the gelation of the system even at a relatively low $C_{\mathrm{AAm}}$. In fact, by adding $1 \mathrm{~mol} \%$ cross-linker $N, N^{\prime}$-methylene bisacrylamide (MBAA) to the precursor solution that forms physical gels in phase I after polymerization, the structure of the gel transforms to phase II (Figure S7). With the same $C_{\mathrm{DGI}}$ and $C_{\mathrm{AAm}}$, the chemical gels showed lower swelling ratio in phase II than the physical gels due to the enhanced cross-linking.

The tensile performances of the swollen PDGI/PAAm gels along the surface of the sheetshaped samples are shown in Figure 4A. In phase I, the gels showed high modulus of 0.06 MPa, a relatively high fracture stress of $0.04 \mathrm{MPa}$ and fracture strain of $2 \sim 2.5$. The high modulus is associated with the rigid bilayers that are in continuous foam structure. In phase II, the gels showed similar modulus along the bilayer directions with that in phase I but much higher fracture stress $(0.10 \sim 0.18 \mathrm{MPa})$ and fracture strain $(18 \sim 22)$. The samples of phase II also showed a well-defined yielding point at a strain of 0.30 , indicating that above this strain the 
unidirectionally aligned continuous bilayers started to rupture. Furthermore, the gels in phase I and II show full self-recovery property below certain tensile strain (Figure 4B, C, D).
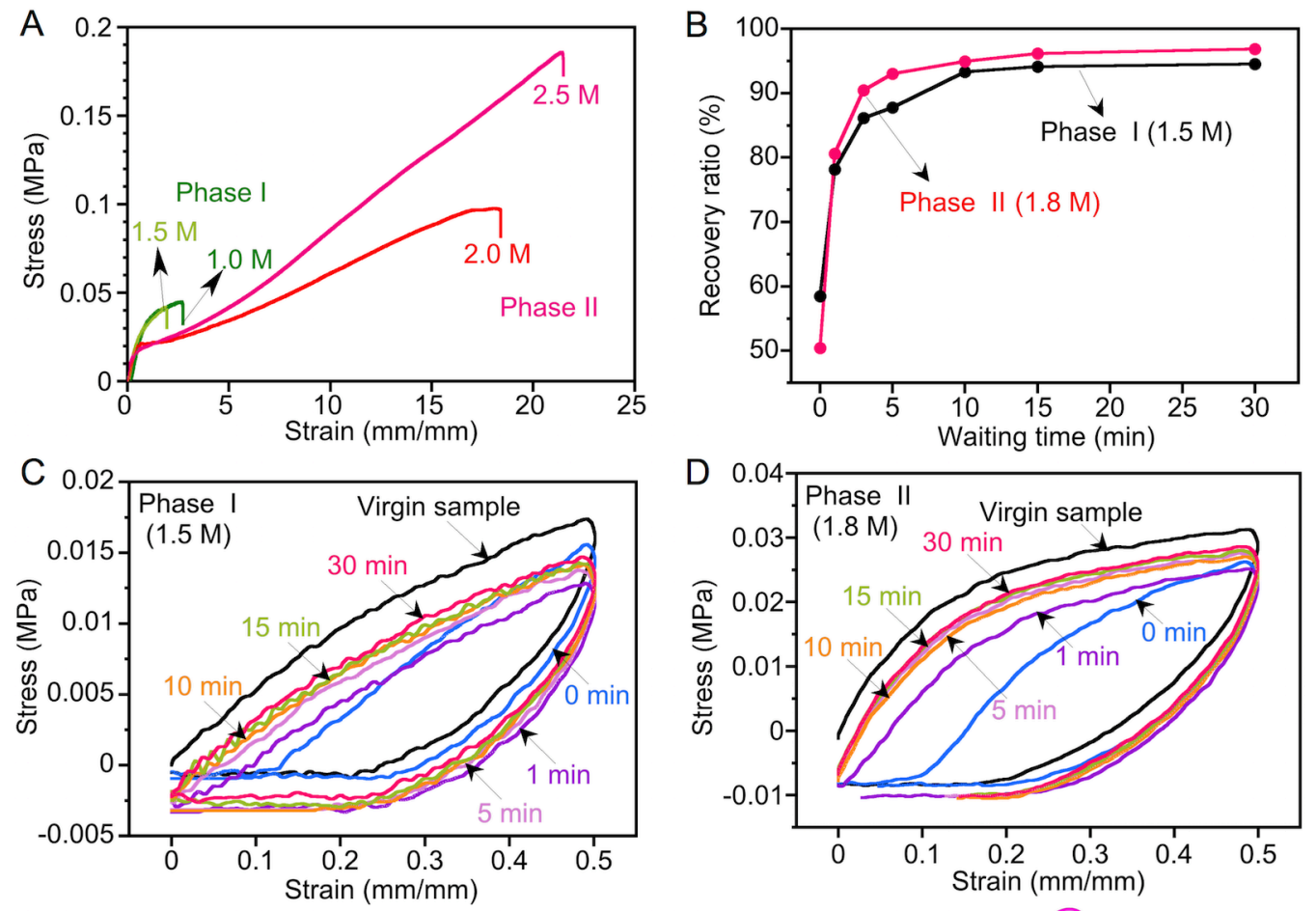

$E$

F
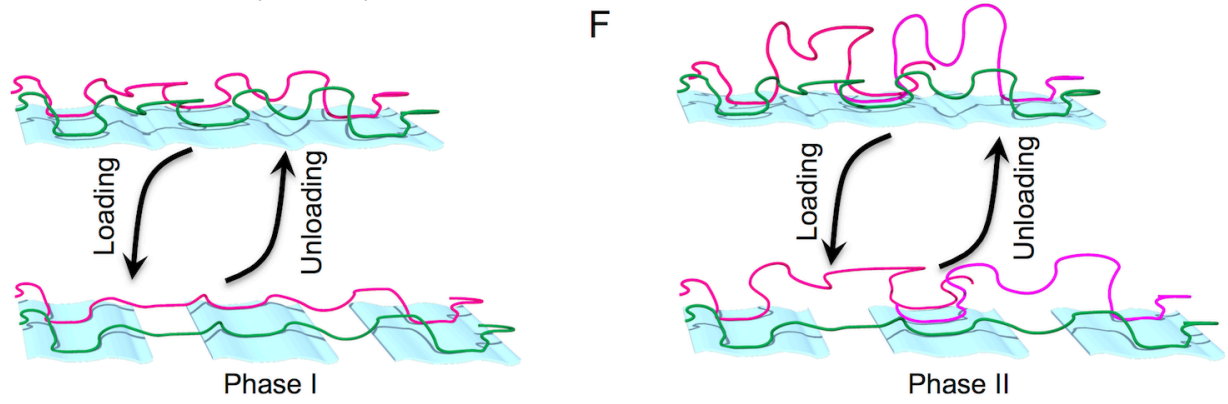

Figure 4. Mechanical properties of the PDGI/PAAm hydrogels in phase I and II. (A) Tensile strain-stress curves of the swollen PDGI/PAAm physical hydrogels. The samples were prepared at $C_{\mathrm{DGI}}=0.1 \mathrm{M}$ and different $C_{\mathrm{AAm}}$, and were elongated in the direction parallel to the sample surface at a stretching velocity of $100 \mathrm{~mm} / \mathrm{min}$ (strain rate: $0.14 \mathrm{~s}^{-1}$ ). (B) Waiting time dependence of the recovery ratio (area ratio of the second hysterisis loop to the first) at a tensile 
strain of 0.5. (C, D) Typical recovery behaviors of the PDGI/PAAm hydrogels in phase I (C) and phase II (D) by cyclic tensile tests performed at various waiting times between the first and second loading cycles. (E, F) Explanation of the self-healing behaviors of the hydrogels in phase I (E) and phase II (F) due to reversible hydrophobic association and elasticity of polymer chains that adsorbed on the bilayers with self-similar feature. For all of the samples, $C_{\text {DGI }}=0.1 \mathrm{M}$, numbers in the figures are $C_{\mathrm{AAm}}$.

Weakly cross-linked PAAm hydrogels measured in the similar condition $\left(C_{\mathrm{AAm}}=2 \mathrm{M}\right.$, crosslinker density $=0.1 \mathrm{~mol} \%$ ) are mechanically weak (fracture stress $=20 \mathrm{kPa}$ ) like common hydrogels. ${ }^{30}$ The physical PDGI/PAAm hydrogels are much stronger, and can dissipate large amount of energy at fracture. Compared with the chemical PDGI/PAAm hydrogel (PAAm crosslinker density: $0.1 \%$ mol\%), the modulus and strain of the physical hydrogels in phase II are similar, but the fracture stress is lower. ${ }^{30}$ The work of extension, which is a parameter to characterize the toughness of the soft material, of the physical gel can reach $2.05 \times 10^{6} \mathrm{~kJ} / \mathrm{m}^{3}$. This is in the same order of tough DN hydrogels, so this gel is also tough although much softer than the DN gels. ${ }^{42}$

The strength of these gels are owing to the rupture of the hydrophobic bonds of bilayers that serves as sacrificial bonds to dissipate mechanical energy. ${ }^{30}$ Since the hydrophobic association between PDGI bilayers are reversible, ${ }^{30}$ and the elasticity of the adsorbed polymer layers remember the initial shape, the ruptured bilayers can self-heal after removal of the stress. Here, the PAAm chain adsorbed within the same bilayer serves as the driving force to reform the ruptured bilayers (Figure 4E, F). As the polymer adsorption has a self-similar structure, ${ }^{34}$ lower PAAm adsorption gives only smaller mesh size of the adsorbed layer. So a thin adsorbed layer 
has smaller extensibility than a thick adsorbed layer (Figure 4E, F). This explains why the gels in phase I are broken at relatively small strain while the gels in phase II sustain very large deformation.

\section{CONCLUSIONS}

The results indicate that the adsorption of PAAm on PDGI lipid bilayers leads to the formation of physical hydrogels, whereupon the amount of polymer adsorption triggers the super structure of the gel through the excluded volume effect. The hydrophobic associations of bilayers serve as sacrificial bonds to dissipate mechanical energy, and the self-similar adsorption of polymer imparts elasticity to the gel. As a result, the hydrogels can be toughened by both the isotropic foam structure (phase I) and the anisotropic lamellar structure (phase II) and exhibit self-healing. This work is helpful to design tough materials with specific macroscopic structures.

Besides, lipid bilayers are widely existent in organisms acting as structural comportments of cell and organelle membranes. The deformation of cells strongly correlates to their functions. For example, pathological changes especially for cancer, always accompany with large cell deformation. ${ }^{43}$ This work also gives new insight to understand the dynamic deformation mechanism of biological cells from the viewpoint of the interaction between phospholipid bilayers and the adjacent cytoskeleton of the cells.

\section{EXPERIMENTAL SECTION}

Physical Gel Preparation. The precursor solution of the physical hydrogels was prepared from an aqueous solution of $0 \sim 0.2 \mathrm{M} \mathrm{DGI,} 0 \sim 3.5 \mathrm{M} \mathrm{AAm}, 0.025$ mol\% sodium dodecyl sulfate (SDS) (in relative to DGI) and $0.1 \mathrm{~mol} \%$ initiator irgacure 2959 (in relative to total 
monomers). The precursor solution was allowed to stand in a water bath for $3 \mathrm{~h}$ at $55^{\circ} \mathrm{C}$ to dissolve DGI powders and form lamellar bilayers of DGI. Then the solution was transferred to a glove box filled with argon to remove the dissolved oxygen. Before polymerization, the solution was injected into a reaction cell of $0.5 \mathrm{~mm}$ in thickness, $90 \mathrm{~mm}$ in length, and $12 \mathrm{~mm}$ in width at a high injection rate to apply a strong shear flow $(\sim 5 \mathrm{~cm} / \mathrm{s})$. The cell was made by two pieces of parallel glass sandwiched by a $0.5 \mathrm{~mm}$ thick silicone spacer. Thus, the DGI molecules in the precursor solution form lamellar phase with the bilayer unidirectionally aligned along the glass walls of the cell. The polymerization was performed under UV light irradiation for $8 \mathrm{~h}$. The whole process in the glove box was maintained at about $50^{\circ} \mathrm{C}$, above the gel-liquid crystalline phase transition temperature $\left(43^{\circ} \mathrm{C}\right)$ of the DGI bilayers. In the above conditions, as the DGI molecules form lamellar bilayers, both DGI and AAm were homo-polymerized by the simultaneous polymerization. ${ }^{33}$ As a comparison, the sole DGI solution and the sole AAm solution were also polymerized individually by the same way with applying the same shear flow.

Swelling Ratio Measurement. After polymerization, the sheet-shaped samples were cut into small pieces of $85 \mathrm{~mm}$ in length and $10 \mathrm{~mm}$ in width, and immersed in water for at least one week to reach equilibrium. The thickness $\left(t_{\mathrm{i}}\right)$, length $\left(l_{\mathrm{i}}\right.$, parallel to shear flow), and width $\left(w_{\mathrm{i}}\right.$, perpendicular to shear flow) of the samples were measured using vernier caliper. Here, the subscript $\mathrm{i}=0,1$, representing for the as-prepared gels, and the water-swollen gels.

Mechanical Characterization. Tensile stress-strain properties of the samples swollen in water were analyzed with a commercial test machine (Tensilon RTC-1310A Orientec Co.). Prior to the test, the sheet-shaped samples of thickness $t$ were cut into dumbbell shape standardized size by the gel cutter (JIS-K6251-7) in a dimension of gauge length $12 \mathrm{~mm}$ and width $2 \mathrm{~mm}$ (Figure S8). The tensile elongation was performed along the sheet direction of the gels. The 
stretching velocity was maintained as $100 \mathrm{~mm} / \mathrm{min}$, corresponding to a strain rate of $0.14 \mathrm{~s}^{-1}$. The moduli of samples along the sheet direction were directly calculated from the initial slope of the tensile stress-strain curves within $10 \%$ elongation. Cyclic tensile tests at various waiting times between the first and second loading cycles were performed in a water bath at room temperature to prevent water from evaporating from the samples.

\section{ASSOCIATED CONTENT}

\section{Supporting Information.}

Additional results and discussions about second-derivative Fourier transform infrared (FTIR) spectroscopic analysis, isotropic/anisotropic swelling, structure transition condition and swelling behavior in DMSO. This material is available free of charge via the Internet at http://pubs.acs.org.

\section{AUTHOR INFORMATION}

\section{Corresponding Author}

*(J.P.G.) Email: gong@mail.sci.hokudai.ac.jp.

\section{Present Addresses}

$\S$ Present addresses: Department of Chemistry, University of Dhaka, Dhaka 1000, Bangladesh (M.A.H.); National institute for Materials Science, 1-2-1 Sengen, Tsukuba, Ibaraki 305-0047, Japan (Y.Y.).

\section{Author Contributions}


X.L., T.K. and J.P.G. conceived the idea. X.L. performed the experiments and wrote the paper. X.L., R.T. and J.P.G. designed the schematic diagram. R.T., M.A.H., Y.Y, T.N. and J.P.G. contributed to the data analysis. J.P.G. supervised the research. X.L. and J.P.G. wrote the manuscript.

\section{Notes}

The authors declare no competing financial interest.

\section{ACKNOWLEDGMENT}

This research was financially supported by a Grant-in-Aid for Scientific Research (S) (No. 124225006) from Japan Society for the Promotion of Science (JSPS).

\section{ABBREVIATIONS}

DGI, dodecyl glyceryl itaconate; AAm, acrylamide; PAAm, polyacrylamide; PDGI, poly(dodecyl glyceryl itaconate); DMSO, dimethyl sulfoxide; SDS, sodium dodecyl sulfate.

\section{REFERENCES}

(1) Gong, J. P.; Katsuyama, Y.; Kurokawa, T.; Osada, Y. Adv. Mater. 2003, 15, 1155-1158.

(2) Sun, J.-Y.; Zhao, X.; Illeperuma, W. R. K.; Chaudhuri, O.; Oh, K. H.; Mooney, D. J.;

Vlassak, J. J.; Suo, Z. Nature 2012, 489, 133-136.

(3) Sun, T. L.; Kurokawa, T.; Kuroda, S.; Ihsan, A. B.; Akasaki, T.; Sato, K.; Haque, M. A.;

Nakajima, T.; Gong, J. P. Nat. Mater. 2013, 12, 932-7.

(4) Shull, K. R. Nature 2012, 489, 36-37.

(5) Calvert, P. Adv. Mater. 2009, 21, 743-756. 
(6) Fukuda, J.; Khademhosseini, A.; Yeo, Y.; Yang, X.; Yeh, J.; Eng, G.; Blumling, J.; Wang,

C.-F.; Kohane, D. S.; Langer, R. Biomaterials 2006, 27, 5259-5267.

(7) Stamatialis, D. F.; Papenburg, B. J.; Gironés, M.; Saiful, S.; Bettahalli, S. N. M.;

Schmitmeier, S.; Wessling, M. J. Membr. Sci. 2008, 308, 1-34.

(8) Slaughter, B. V.; Khurshid, S. S.; Fisher, O. Z.; Khademhosseini, A.; Peppas, N. A. Adv.

Mater. 2009, 21, 3307-3329.

(9) Hirst, A. R.; Escuder, B.; Miravet, J. F.; Smith, D. K. Angew. Chem. Int. Ed. 2008, 47, 80028018.

(10) Hall, Matthew S.; Long, R.; Hui, C.-Y.; Wu, M. Biophys. J. 2012, 102, 2241-2250.

(11) Shiraki, Y.; Akimoto, A. M.; Miyata, T.; Yoshida, R. Chem. Mater. 2014, 26, 5441-5443.

(12) Chan, E. P.; Walish, J. J.; Urbas, A. M.; Thomas, E. L. Adv. Mater. 2013, 25, 3934-47.

(13) Takahashi, R.; Wu, Z. L.; Arifuzzaman, M.; Nonoyama, T.; Nakajima, T.; Kurokawa, T.;

Gong, J. P. Nat. Commun. 2014, 5, 4490-4496.

(14) White, S. H.; Wimley, W. C. Ann. Rev. Biophys. Biomol. Struct. 1999, 28, 319-365.

(15) Weber, T.; Zemelman, B. V.; McNew, J. A.; Westermann, B.; Gmachl, M.; Parlati, F.;

Söllner, T. H.; Rothman, J. E. Cell 1998, 92, 759-772.

(16) Discher, B. M.; Won, Y.-Y.; Ege, D. S.; Lee, J. C.-M.; Bates, F. S.; Discher, D. E.;

Hammer, D. A. Science 1999, 284, 1143-1146.

(17) Hajduk, D. A.; Kossuth, M. B.; Hillmyer, M. A.; Bates, F. S. J. Phys. Chem. B 1998, 102, $4269-4276$.

(18) Hillmyer, M. A.; Bates, F. S.; Almdal, K.; Mortensen, K.; Ryan, A. J.; Fairclough, J. P. A. Science 1996, 271, 976-978.

(19) Won, Y. Y.; Davis, H. T.; Bates, F. S. Science 1999, 283, 960-963. 
(20) Yu, K.; Eisenberg, A. Macromolecules 1998, 31, 3509-3518.

(21) Warriner, H. E.; Idziak, S. H. J.; Slack, N. L.; Davidson, P.; Safinya, C. R. Science 1996, $271,969-973$.

(22) Eng, G.; Lee, B. W.; Parsa, H.; Chin, C. D.; Schneider, J.; Linkov, G.; Sia, S. K.; VunjakNovakovic, G. Proc. Natl. Acad. Sci. USA 2013, 110, 4551-4556.

(23) Bian, L.; Guvendiren, M.; Mauck, R. L.; Burdick, J. A. Proc. Natl. Acad. Sci. USA 2013, $110,10117-10122$.

(24) Kopecek, J.; Yang, J. Y. Angew. Chem. Int. Ed. 2012, 51, 7396-7417.

(25) Chen, P.; Yang, J. J.; Li, S. S.; Wang, Z.; Xiao, T. Y.; Qian, Y. H.; Yu, S. H. Nano Energy 2013, 2, 249-256.

(26) Park, Y.; Hashimoto, C.; Hashimoto, T.; Hirokawa, Y.; Jung, Y. M.; Ozaki, Y. Macromolecules 2013, 46, 3587-3602.

(27) Liu, M.; Ishida, Y.; Ebina, Y.; Sasaki, T.; Hikima, T.; Takata, M.; Aida, T. Nature 2015, $517,68-72$.

(28) Haque, M. A.; Kamita, G.; Kurokawa, T.; Tsujii, K.; Gong, J. P. Adv. Mater. 2010, 22, $5110-5114$

(29) Yue, Y. F.; Haque, M. A.; Kurokawa, T.; Nakajima, T.; Gong, J. P. Adv. Mater. 2013, 25, 3106-10.

(30) Haque, M. A.; Kurokawa, T.; Kamita, G.; Gong, J. P. Macromolecules 2011, 44, 89168924.

(31) Yue, Y.; Kurokawa, T.; Haque, M. A.; Nakajima, T.; Nonoyama, T.; Li, X.; Kajiwara, I.; Gong, J. P. Nat. Commun. 2014, 5, 4659-4666.

(32) Naitoh, K.; Ishii, Y.; Tsujii, K. J. Phys. Chem. 1991, 95, 7915-7918. 
(33) Ozawa, J.; Matsuo, G.; Kamo, N.; Tsujii, K. Macromolecules 2006, 39, 7998-8002.

(34) de Gennes, P. G. Macromolecules 1981, 14, 1637-1644.

(35) de Gennes, P. G. Macromolecules 1982, 15, 492-500.

(36) Stuart, M. A. C.; Waajen, F. H. W. H.; Cosgrove, T.; Vincent, B.; Crowley, T. L. Macromolecules 1984, 17, 1825-1830.

(37) de Gennes, P. G. Adv. Colloid Interface Sci. 1987, 27, 189-209.

(38) Baker, J. A.; Pearson, R. A.; Berg, J. C. Langmuir 1989, 5, 339-342.

(39) Onsager, L. Ann. N. Y. Acad. Sci. 1949, 51, 627-659.

(40) Landau, L. D.; Lifshitz, E. M., Statistical Physics. Pergamon, London: 1958.

(41) de Gennes, P.-G., Scaling Concepts in Polymer Physics. Cornell University Press: Ithaca, NY: 1979.

(42) Gong, J. P. Soft Matter 2010, 6, 2583-2590.

(43) Porporato, Paolo E.; Payen, Valéry L.; Pérez-Escuredo, J.; De Saedeleer, Christophe J.;

Danhier, P.; Copetti, T.; Dhup, S.; Tardy, M.; Vazeille, T.; Bouzin, C.; Feron, O.; Michiels, C.; Gallez, B.; Sonveaux, P. Cell Reports 2014, 8, 754-766. 
for Table of Contents use only

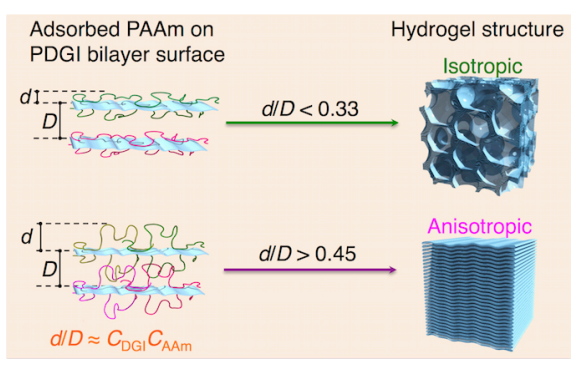

Title: Polymer Adsorbed Bilayer Membranes Form Self-healing Hydrogels with Tunable Superstructure

Authors: Xufeng Li, Takayuki Kurokawa, Riku Takahashi, Md. Anamul Haque, Youfeng Yue, Tasuku Nakajima, and Jian Ping Gong* 